\title{
Acurácia da Histeroscopia na Avaliação da Cavidade Uterina em Pacientes com Sangramento Uterino Pós-Menopausa
}

\author{
Accuracy of Hysteroscopy in the Evaluation of the Uterine Cavity in Patients \\ with Postmenopausal Uterine Bleeding
}

Maria Karenina N. Machado, Hilton Pina, Eliana Matos

\begin{abstract}
RESUMO
Objetivo: investigar a acurácia da histeroscopia como método de estudo da cavidade uterina de pacientes com sangramento na pós-menopausa.

Método: trata-se de estudo transversal, que consistiu na avaliação de 78 prontuários de pacientes menopausadas com queixa de sangramento, no periodo de janeiro de 2000 a junho de 2002 no Centro Estadual de Oncologia do Estado da Bahia, submetidas à histeroscopia com biópsia de endométrio. Os achados histeroscópicos foram classificados como benignos (miomas, pólipos, atrofia, normal) e suspeitos (espessamento, hiperplasias e câncer) e os achados histopatológicos como benignos (miomas, pólipos, atrofia, hiperplasia típica) e malignos (hiperplasia atipica e câncer). Os resultados da histeroscopia foram comparados com os exames histopatológicos.

Resultados: em relação aos achados suspeitos (espessamento, hiperplasias e câncer), a sensibilidade da histeroscopia foi de 85,7\% e a especificidade de 88,7\%, quando comparados à histopatologia. O valor preditivo positivo foi de 42,8\% e o negativo 98,4\%. A razão de probabilidades do teste positivo e a razão de probabilidades do teste negativo foram respectivamente 7,6 e 0,16. A acuidade da histeroscopia foi de 88,4\% e o indice kappa 0,5. Conclusão: a histeroscopia isoladamente não apresentou, no presente estudo, acurácia aceitável, reforçando o conceito de que sua principal vantagem é dirigir a biópsia, devendo sempre estar associada ao diagnóstico histopatológico.
\end{abstract}

PALAVRAS-CHAVE: Histeroscopia. Pós-Menopausa. Endométrio: carcinoma. Endométrio: avaliação.

\section{Introdução}

As mulheres menopausadas representam hoje parcela significativa da população feminina. A expectativa de vida no Brasil é de 72,5 anos (IBGE, 2001), podendo ultrapassar os 80 anos em países de primeiro mundo. Estima-se, portanto, que as mulheres passarão mais de um terço de suas vidas na pós-menopausa ${ }^{1}$.

Centro Estadual de Oncologia do Estado da Bahia

Correspondência:

Maria Karenina N. Machado

Centro Médico Salvador, Sala 11

Av. Juracy Magalhães Júnior, 2490 - Rio Vermelho

41940-060 - Salvador - BA

Tel: (71) 351-5302/ 359-3900

e-mail: ak_machado@ibest.com.br
São diversas as causas de sangramento proveniente do útero. Eles podem ocorrer em qualquer idade, sendo resultado de problemas relacionados à anovulação, lesões anatômicas benignas ou malignas, gravidez ou distúrbios hormonais. Aproximadamente $5 \%$ a $33 \%$ das mulheres que procuram clínicas ginecológicas têm como queixa principal o sangramento uterino ${ }^{2}$, e, destas mulheres, $69 \%$ encontram-se na pós-menopausa ${ }^{3}$. Embora o sangramento pós- menopausa possa ser devido a outras condições clínicas, o câncer endometrial é a causa mais importante ${ }^{4}$.

Tradicionalmente o sangramento pós-menopausa tem sido investigado por meio de biópsia de endométrio ${ }^{2}$. Antes de 1982, a avaliação diagnóstica era rotineiramente realizada pela curetagem uterina. 
Entretanto este procedimento tem resultados falso-negativos de 2 a $10 \%{ }^{5}$, e menos da metade da cavidade uterina é efetivamente curetada em $60 \%$ dos casos $^{6}$. Os miomas submucosos e pólipos endometriais muitas vezes não são diagnosticados nas biópsias, tendo índice de falha de até $50 \%{ }^{7}$.

A ultra-sonografia transvaginal é essencial para avaliação das causas de sangramento pósmenopausa, avaliação do endométrio na reposição hormonal e qualquer alteração na cavidade pélvica. Porém, não permite a diferenciação inequivoca das imagens endometriais entre pólipos, hiperplasias e fenômenos proliferativos resultantes de terapia de reposição hormonal ${ }^{8}$, assim como na determinação da localização submucosa ou intramural de um mioma9 .

A histeroscopia associada à histopatologia é excelente método para a avaliação da cavidade uterina, seja em casos de sangramento ou para investigação de pacientes inférteis. É exame dinâmico que pode ser realizado ambulatorialmente e sua principal vantagem é permitir a biópsia dirigida da área de maior suspeição ${ }^{8}$.

O objetivo deste trabalho foi investigar a acurácia da histeroscopia como método de estudo da cavidade uterina no sangramento pós-menopausa, comparando as imagens obtidas com os resultados histopatológicos.

\section{Pacientes e Métodos}

Foi realizado estudo transversal com 78 pacientes submetidas à histeroscopia no período de janeiro de 2000 a junho de 2002 no Centro Estadual de Oncologia do Estado da Bahia (CICAN). Os critérios básicos para a inclusão no estudo foram: mulheres menopausadas que tinham como queixa principal sangramento uterino e que foram submetidas à histeroscopia com biópsia de endométrio. Os critérios de exclusão foram: presença de doenças cervicais, mulheres histerectomizadas e mulheres na pré-menopausa. As pacientes poderiam ou não estar sob uso de qualquer esquema de terapia de reposição hormonal. As pacientes cujas histeroscopias não foram realizadas por qualquer motivo (por ex., estenose cervical) foram excluídas do estudo. Não valorizamse neste estudo resultados de exames prévios.

O projeto desta pesquisa foi encaminhado ao Comitê de Ética em Pesquisa da Maternidade Climério de Oliveira/Curso de Pós-graduação em Medicina e Saúde.

A média de idade das pacientes foi de 63,4 , desvio padrão de 9,3 anos, com mediana de 64,0 anos. O número médio de gestações foi de $7,1 \pm 4,6$ com mediana de 6,0 gestações. Um quarto das pacientes que procuraram o Serviço eram provenientes da capital, $70,2 \%$ do interior e 4,3\% de outros estados. Em relação ao grau de escolaridade, $61,6 \%$ das pacientes tinham $1^{\circ}$ grau incompleto, $9,6 \% 2^{\circ}$ grau completo e $28,8 \%$ eram analfabetas. Os outros niveis de instrução não foram encontrados. Somente 7,6\% das pacientes faziam uso de terapia de reposição hormonal.

As amostras para estudo histopatológico foram obtidas por meio de biópsias realizadas com a cânula de Karman. A histeroscopia foi realizada sob sedação com o micro-histeroscópio de Hamou II, marca Storz, com óptica de 4,0 mm de diâmetro, com ângulo de 30 graus e camisa de 5,0 mm, com canal para passagem do meio distensor. Para a distensão da cavidade uterina utilizou-se meio gasoso $\left(\mathrm{CO}_{2}\right)$ com controle eletrônico de fluxo e pressão, insuflado com o micro-histeroflator de Hamou (Storz). O exame foi realizado por dois observadores com mais de dois anos de experiência e a anatomia patológica por três patologistas também pertencentes ao Serviço. Os achados histeroscópicos foram classificados como: benignos (pólipos, miomas, atrofia, normal) e suspeitos (espessamento, hiperplasias, câncer), e os achados histopatológicos como: benignos (pólipos, miomas, hiperplasia típica, atrofia, normal) e malignos (hiperplasia atípica, câncer). Os resultados inconclusivos da biópsia foram classificados como atróficos. A anatomia patológica foi considerada o padrão-ouro.

Para análise dos dados foram utilizados os procedimentos usuais da estatística descritiva, tais como o cálculo de freqüências simples ou relativas, algumas medidas de tendência central e de dispersão. Para a avaliação da acurácia foram calculados os indicadores de validade: sensibilidade (S), especificidade (E), valor preditivo positivo (VPP), valor preditivo negativo (VPN), razão de probabilidades do teste positivo (RPP), razão de probabilidades do teste negativo (RPN), acuidade (AC) e o índice kappa. O programa estatístico utilizado foi o Statistical Package for the Social Sciences (SPSS) ${ }^{10}$.

As pacientes incluídas no estudo constituíram uma amostra, mas esta não foi selecionada aleatoriamente. Assim, considerou-se sem sentido calcular-se inferência estatística. Contudo, não acreditamos que as 78 pacientes estudadas tenham características clínico-epidemiológicas diferentes daquelas atendidas antes ou depois do período de coleta dos dados do presente estudo. Desta forma, os resultados obtidos na amostra investigada poderão ser generalizados para toda a clientela do CICAN, com base neste critério não estatístico. 


\section{Resultados}

Os achados histeroscópicos mostraram freqüência de $42,3 \%$ para pólipo endometrial, 14,1\% para hiperplasia, $2,6 \%$ para espessamento endometrial, 3,8\% para câncer e 2,6\% para mioma. Os achados histopatológicos mostraram freqüência de 25,6\% para pólipo endometrial, 2,6\% para mioma, $6,4 \%$ para hiperplasia típica e $9,0 \%$ para câncer (Figura 1).

Em relação aos achados suspeitos a histeroscopia mostrou sensibilidade de $85,7 \%$, especificidade de $88,7 \%$, VPP de $42,8 \%$, VPN de $98,4 \%$. RPP e a RPN foram respectivamente de 7,6 e 0,16. A probabilidade de a histeroscopia classificar corretamente os indivíduos como negativos é $84,0 \%$ maior do que a probabilidade de a histeroscopia classificar incorretamente os indivíduos como negativos. A acuidade da histeroscopia foi de $88,4 \%$ e o indice kappa 0,5 (Tabela 1).

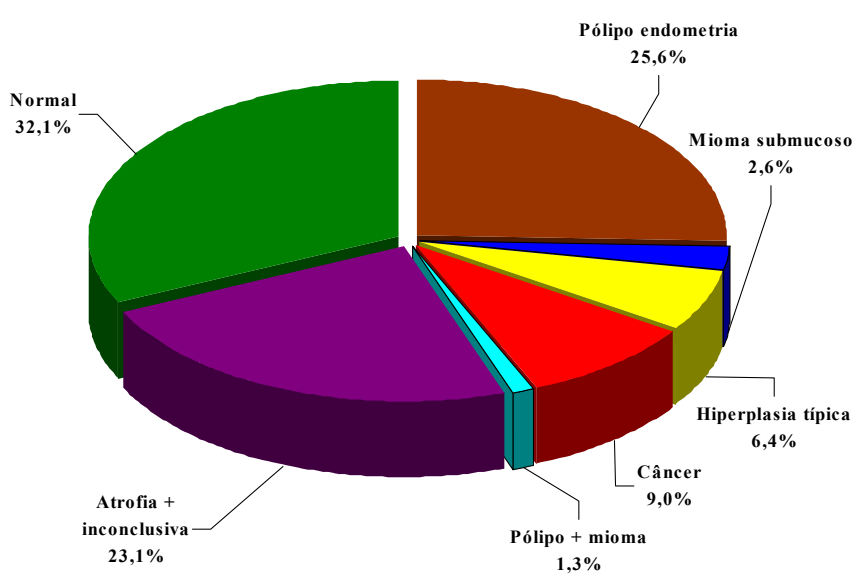

Figura 1- Freqüência de achados histopatológicos.

Tabela 1 - Valores de S, E, VPP, VPN, RPP, RPN, AC e índice kappa da histeroscopia em relação aos diagnósticos histopatológicos.

\begin{tabular}{lcccccccc}
\hline Anomalias & $\mathbf{S}$ & $\mathbf{E}$ & VPP & VPN & RPP & RPN & $\begin{array}{c}\text { AC } \\
(\%)\end{array}$ & $\begin{array}{c}\text { Índice } \\
\text { kappa }\end{array}$ \\
\hline Hiperplasia & 60,0 & 91,7 & 33,3 & 97,1 & 7,3 & 0,4 & 89,7 & 0,3 \\
Pólipo & 94,4 & 75,0 & 53,1 & 97,8 & 3,7 & 0,07 & 79,4 & 0,4 \\
Mioma & 50,0 & 98,6 & 50,0 & 98,6 & 38,4 & 0,5 & 97,4 & 0,4 \\
Câncer & 42,8 & 100,0 & 100,0 & 94,6 & 42 & 0,5 & 94,8 & 0,5 \\
\hline
\end{tabular}

S (sensibilidade), E (especificidade), VPP (valor preditivo positivo), VPN (valor preditivo negativo), RPP (razão de probabilidade do teste positivo), RPN (razão de probabilidade do teste negativo), AC (acuidade).

Para os sete casos de câncer, a sensibilidade da histeroscopia foi de $42,8 \%$ e a especificidade de 100,0\%. Dos 18 casos com pólipo endometrial, a histeroscopia diagnosticou corretamente dezessete casos. Os níveis de sensibilidade e especificidade para pólipo endometrial foram respectivamente $94,4 \%$ e $75,0 \%$. Em relação aos miomas submucosos a histeroscopia mostrou nível de sensibilidade de 50,0\% e especificidade de $98,6 \%$ (Tabela 1).

\section{Discussão}

Os avanços que têm sido incorporados à histeroscopia na última década têm permitido maior segurança no procedimento, ademais da obtenção de imagens mais nítidas e pré-definidas na suspeição diagnóstica, o que tem melhorado os resultados oriundos das biópsias endometriais. A histeroscopia está indicada em todas as situações clínicas nas quais a visualização da cavida- de uterina possa fornecer subsídios para um diagnóstico preciso e conseqüente conduta terapêutica. A avaliação do sangramento uterino anormal e a investigação do fator uterino na infertilidade constituem as indicações mais freqüentes para a realização da histeroscopia.

Vale ressaltar, entretanto, que com os avanços tecnológicos introduzidos na área da ultra-sonografia vem aumentando o número de indicações em pacientes assintomáticas que apresentam imagens de alterações intracavitárias detectadas por esse método.

$\mathrm{Na}$ pesquisa do sangramento uterino é fundamental diferenciar as pacientes pré e pós-menopausadas. Nessas etapas da vida da mulher, as causas determinantes do sangramento variam, principalmente, em função dos niveis séricos dos hormônios esteróides ovarianos. Na pré-menopausa, em quase metade das pacientes, a etiologia é funcional. Na pós-menopausa o prognóstico é mais grave e as causas residem, na maioria das vezes, numa doença orgânica, sobressaindo-se os pólipos, os miomas submucosos, hiperplasias endome- 
triais e, mais raramente, o carcinoma do endométrio. Neste grupo de mulheres, o procedimento histeroscópico deve ser sempre complementado com a feitura de biópsia para a realização de exame histopatológico.

A alta incidência de carcinoma endometrial em mulheres com sangramento pós-menopausa reforça a necessidade de procedimento diagnóstico mais acurado ${ }^{11}$.

A histeroscopia associada à biópsia dirigida de endométrio é exame simples, rápido, seguro e com baixa incidência de complicações ${ }^{12}$, que vem se firmando como método promissor para este fim, principalmente nos casos de processos focais do endométrio como os pólipos e miomas submucosos.

Pólipos e miomas podem, eventualmente, não ser diagnosticados pelos exames tradicionais (curetagem uterina e ultra-sonografia transvaginal) e constituir causa de sangramento recorrente $^{13}$. Em nosso estudo a histeroscopia mostrou grande eficiência no diagnóstico de pólipos $(94,4 \%)$, concordando com dados da literatura ${ }^{14}$.

Muitos estudos têm mostrado a alta acurácia diagnóstica da histeroscopia nos casos de câncer de endométrio ${ }^{3}$. Mortakis e Mavrelos ${ }^{15}$ citam a histeroscopia combinada com biópsia como padrãoouro no diagnóstico de câncer de endométrio, com $100 \%$ de sensibilidade. Souza et al. ${ }^{13}$, em 88 histeroscopias, tiveram 9 casos de câncer (13\%), com niveis de sensibilidade de $88,9 \%$, especificidade de $98,3 \%$, valor preditivo positivo de $88,9 \%$ e valor preditivo negativo de 98,3\%.

A baixa sensibilidade da histeroscopia para o diagnóstico do câncer de endométrio no presente estudo pode ser atribuida aos estágios iniciais da neoplasia, os quais podem não redundar em suspeita diagnóstica e exigir complementação anatomopatológica a fim de se obter um diagnóstico definitivo.

Este exame é também considerado método de excelência na distinção entre mioma submucoso e pólipo endometrial ${ }^{16}$. Entretanto, no presente estudo, a histeroscopia mostrou baixa sensibilidade para miomas submucosos, o que pode ser atribuido a miomas pequenos, calcificados, os quais são mais freqüentes na faixa etária selecionada.

Outro fato igualmente importante a ser analisado é a maneira de realização da biópsia de endométrio através da cânula de Karman, a qual pode ter contribuido para os baixos indices de sensibilidade nos casos de miomas submucosos (50\%), câncer de endométrio (42,8\%) e hiperplasia $(60 \%)$. A biópsia orientada pela histeroscopia pode ter provocado superestimativa dos indicadores de validade calculados, reforçando a idéia de que a histeroscopia deve sempre ser associada à histopatologia.

Concluimos que, embora isoladamente a histeroscopia não tenha apresentado acurácia aceitável, o exame quando associado à biópsia é método importante na avaliação da cavidade uterina, com boa acurácia diagnóstica para pólipos, afastando com excelente validade o câncer endometrial.

\section{ABSTRACT}

Purpose: to investigate the accuracy of hysteroscopy as a method for the evaluation of the uterine cavity in women with postmenopausal bleeding.

Methods: a cross-sectional study that consisted of the evaluation of 78 women with postmenopausal bleeding submitled to histeroscopy and directed biopsy in the period from January 2000 to June 2002 in the Bahia State Oncology Center. Hysteroscopy findings were classified as benign (leiomyoma, polyp, atrophy, normal) and suspect (hyperplasia, thickening, cancer) and the histopathologic findings as benign (leiomyoma, polyp, non-atypical hyperplasia, atrophy) and malignant (cancer and atypical hyperplasia). The results of hysteroscopy were compared with the pathologic findings.

Results: in relation to the suspect results (thickening, hyperplasia and cancer) hysteroscopy sensitivity and specificity were 85.7 and $88.7 \%$, respectively. Positive and negative predictive values were 42.83 and $98.4 \%$. Likelihood ratios of positive and negative tests were 7.6 and 0.16 . Accuracy was $88.4 \%$ and kappa index, 0.5.

Conclusion: hysteroscopy alone did not show an acceptable accuracy in the study, reinforcing the idea that its main advantage is to direct the biopsy, and it must always be associated with the histological diagnosis.

KEYWORDS: Hysteroscopy. Menopause. Endometrium: carcinoma. Endometrium: evaluation.

\section{Referências}

1. Marinho R. Climatério. $2^{a}$ ed. Rio de Janeiro: Medsi; 2000. p.410.

2. Briley M, Lindsell DR.The role of ultrasound in the investigation of women with post-menopausal bleeding. Clin Radiol 1998; 53:502-5.

3. Tahir MM, Bigrigg MA, Browning JJ, Brookes ST, Smith PA. A randomised controlled trial comparing transvaginal ultrasound, outpatient hysteroscopy and endometrial biopsy with in patient hysteroscopy and curettage. Br J Obstet Gynaecol 1999; 106:1259-64. 
4. Royal College of Obstetricians and Gynaecologists. Inpatient treatment: $\mathrm{D} \& \mathrm{C}$ in women age 40 or less. London: RCOG; 1994. p.1-4. (Guidelines, 3).

5. Vandendael A, Debois P, van den Bosch T. Sonohysterography in the detection of endometrial pathology. S Afr Med J 1995; 85:1197.

6. Gaucherand P, Piacenza JM, Salle B, Rudigoz RC. Sonohysterography of the uterine cavity: preliminary investigations. J Clin Ultrasound 1995; 23:339-48.

7. Goldstein SR. Saline infusion sonohysterography. Clin Obstet Gynecol 1996; 39:248-58.

8. Bonilla-Musoles F, Raga F, Osborne NG, Blanes J, Coelho F. Three-dimensional hysterosonography for the study of endometrial tumors: comparison with conventional transvaginal sonography, hysterosalpingography, and hysteroscopy. Gynecol Oncol 1997; 65:245-52.

9. Gronlund L, Hertz J, Helm P, Colov NP. Transvaginal sonohysterography and hysteroscopy in the evaluation of female infertility, habitual abortion or metrorrhagia. A comparative study. Acta Obstet Gynecol Scand 1999; 78:415-8.

10.Norusis MJ. Statistical package for the social sciences for Windows: advanced statistics [computer program]. Release 9.0. Chicago (IL): SPSS; 1993.
11.Grimes DA. Diagnostic dilation and curettage: a reappraisal. Am J Obstet Gynecol 1982; 142:1-6.

12.Lasmar R, Barrozo P. Histeroscopia: uma abordagem prática. $1^{a}$ ed. Rio de Janeiro: Medsi; 2002. p.17179.

13. Souza R, Silvestre M, Almeida e Sousa L, et al. Transvaginal ultrasonography and hysteroscopy in postmenopausal bleeding: a prospective study. Acta Obstet Gynecol Scand 2001; 80:856-62.

14.Giusa-Chiferi MG, Gonçalves WJ, Baracat EC, Albuquerque Neto LC, Bortoletto CC, Lima GR. Transvaginal ultrasound, uterine biopsy and hysteroscopy for post-menopausal bleeding. Int $\mathrm{J}$ Gynaecol Obstet 1996; 55:39-44.

15. Mortakis AE, Mavrelos K. Transvaginal ultrasonography and hysteroscopy in the diagnosis of endometrial abnormalities. J Am Assoc Gynecol Laparosc 1997; 4:449-52.

16.Lurain JR. Uterine cancer. In: Berek JS, Adashi EY, Hillard PA, editors. Novak's Gynecology. $12^{\text {th }}$ ed. Baltimore: William \& Wilkins; 1996. p.1057-110.

Recebido em: 25/3/2003 Aceito com modificações em: 25/4/2003

\section{CONCURSO}

PARA OBTENÇÃO DO CERTIFICADO DE ATUAÇÃO NA ÁREA $17 / 11 / 2003$
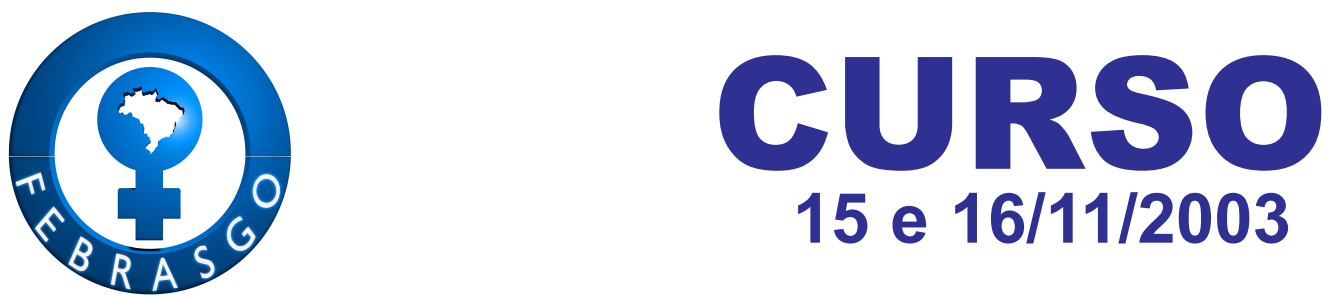

ATUAÇÃO NAS ÁREAS:

VÍDEO-ENDOSCOPIA GINECOLÓGICA

(Laparoscopia e Histeroscopia)

ULTRA-SONOGRAFIA

URODINÂMICA E URETROCISTOSCOPIA

INSCRIÇÕES 11/8/2003 à 12/9/2003

NA FEDERADA DO SEU ESTADO

\section{LOGAl: CENTRO de CONVENĢÕES dE PERNAMBUCO OLINDA/PE}


Laboratório: Sanofi
Anúncio: Dermacyd 